\title{
Delegation und Substitution ärztlicher Aufgaben - Wohin führt der Weg in der Psychiatrie?
}

\author{
Delegation and Substitution of Physicians' Incumbencies - What is the Way in \\ Psychiatry?
}

Autoren

Institut
Hermann Spießl, Helmut Hausner, Göran Hajak, Helmfried E. Klein

Klinik und Poliklinik für Psychiatrie, Psychosomatik und Psychotherapie der Universität am Bezirksklinikum Regensburg
Bibliografie

DOI $10.1055 / \mathrm{s}-2008-1067486$

Psychiat Prax 2008; 35:

265-266

(c) Georg Thieme Verlag KG Stuttgart · New York .

ISSN 0303-4259

Korrespondenzadresse

Priv.-Doz. Dr. med.

Hermann SpießI

Bezirksklinikum Regensburg

Universitätsstraße 84

93053 Regensburg

hermann.spiessl@medbo.de
Aufgrund der Zunahme der Fallzahlen und der Fallschwere wird zukünftig eine vermehrte Nachfrage nach Krankenhausärzten bestehen, auch in der Psychiatrie. Schon heute können Kliniken etwa $25 \%$ der offenen Stellen nicht mehr besetzen. Diese Situation wird sich aufgrund der Altersstruktur der Ärzte in den nächsten 10 Jahren weiter verschlechtern.

Vor diesem Hintergrund hat das Deutsche Krankenhausinstitut (DKI) im Auftrag der Deutschen Krankenhausgesellschaft (DKG) das Projekt „Neuordnung von Aufgaben des ärztlichen Dienstes“ durchgeführt [1], bei dem Entscheidungsträger aus 304 Krankenhäuser befragt wurden. Ziel war es herauszufinden, welche Tätigkeiten des ärztlichen Dienstes von nichtärztlichen Berufsgruppen übernommen werden können und dürfen. Bereits im Gutachten des Sachverständigenrates zur Entwicklung des Gesundheitswesens von 2007 wurde auf die Notwendigkeit einer Neudefinition der Aufgaben verschiedener Berufsgruppen im Gesundheitswesen hingewiesen [2]. Aus juristischer Sicht sei allerdings ein unantastbarer Kernbereich ärztlicher Aufgaben zu benennen, der nicht delegierbar sei. Behandlungsmaßnahmen, die aufgrund ihrer Schwierigkeit, Gefährlichkeit oder wegen Unvorhersehbarkeit etwaiger Reaktionen spezifisches ärztliches Wissen voraussetzten, seien vom Arzt persönlich durchzuführen oder unmittelbar zu überwachen. Tätigkeiten im Kernbereich der Diagnostik, Differenzialdiagnostik und Therapie werden somit weiter nicht delegierbar sein, insbesondere wenn dadurch eine Gefährdung für die Gesundheit des Patienten nicht ausgeschlossen werden kann. Hier wird der bisherige sog. Arztvorbehalt weiter bestehen bleiben, wie er beispielsweise in $\S 15$ Abs.1 SGB V festgeschrieben ist, wonach ärztliche Behandlung auch nur von Ärzten zu erbringen ist und Hilfeleistungen anderer Berufsgruppen von den Ärzten zu verantworten sind.
Welche Aufgaben können nun an nichtärztliches Personal übertragen werden? Das DKI macht diesbezüglich eine Reihe von Vorschlägen, die überwiegend den somatischen Bereich betreffen. Es teilt die Tätigkeiten in kurzfristige, mittelfristige und langfristige übertragbare Aufgaben ein: 1. Kurzfristig übertragbare Tätigkeiten können nach kurzer Einweisung vom nichtärztlichen Personal in gleicher Qualität durchgeführt werden. Beispiele sind: Codierung von Diagnosen, venöse Blutentnahmen, intramuskuläre Injektionen, aber auch intravenöse Injektion von Medikamenten und Anlage einer Venenverweilkanüle.

2. Mittelfristig übertragbare Tätigkeiten erfordern eine umfangreichere Anpassung der Qualifikation. Als Beispiele werden genannt: Nichtärztliche Assistenz bei operativen Eingriffen, Wundpflegemanagement oder Schmerzmanagement.

3. Langfristig übertragbare Tätigkeiten können erst erfolgen, wenn entsprechende rechtliche Normen geändert werden. Als Beispiel wird der Einsatz nichtärztlichen Personals bei Parallelnarkosen in der Anästhesie genannt.

Ziel all dieser Maßnahmen sei laut DKG der notwendige „effizientere Personaleinsatz im Krankenhaus“" [3]. Aus Sicht der DKG könne auch bei einer Neuordnung der ärztlichen Tätigkeiten bei entsprechender Qualifikation des nichtärztlichen Personals - eine kompetente Betreuung der Patienten sichergestellt werden.

Welche Implikationen ergeben sich daraus für die Psychiatrie? Bei Durchsicht des Kataloges der übertragbaren Tätigkeiten [1] zeigt sich, dass viele davon bereits in den psychiatrischen Kliniken gerade vom Pflegepersonal durchgeführt werden (z.B. venöse Blutentnahmen, intramuskuläre und subkutane Injektionen, Ruhe-EKG u.a.). Viele der ansonsten genannten Tätigkeiten sind im Bereich der somatischen Medizin angesiedelt und haben für die Psychiatrie keine Relevanz. 
Darüber hinaus werden von nichtärztlichen Mitarbeitern in einer psychiatrischen Klinik eine Reihe von weiteren Aufgaben übernommen, die in der DKI-Studie gar nicht erfasst wurden: So leiten häufig Pflegekräfte Entspannungsgruppen oder Aktivierungsgruppen. Sie führen die Wachtherapie und Lichttherapie durch und sind Cotherapeuten in Gruppenpsychotherapien (insb. Psychoedukation) und in Angehörigengruppen. Auch das Stationsund Patientenmanagement vom Zeitpunkt der Aufnahme bis zur Entlassung mit Erledigung zahlreicher administrativer Aufgaben erfolgt kompetent durch das Pflegepersonal. Viele der vom DKI vorgeschlagenen Tätigkeiten im Bereich der Administration und Dokumentation werden dadurch bereits abgedeckt. Das mögliche Einsparpotenzial in psychiatrischen Kliniken ist aufgrund der vielerorts bereits praktizierten vielfältigen Einbindung des Pflegepersonals in den gesamten Behandlungsprozess somit wohl eher gering, es sei denn, dass auch die Erhebung von Teilen der Anamnese (z. B. Sozial- und Fremdanamnese) von einer qualifizierten Pflegekraft übernommen wird; hinsichtlich der Erhebung von Basisdaten der Biografie erscheint dies auch möglich und effizient, wenn dies standardisiert erfolgt und zu keiner doppelten Erhebung durch Arzt und Pflegepersonal führt.

Eine sinnvolle Delegation ärztlicher Aufgaben an nichtärztliches Fachpersonal könnte sich im administrativen Bereich ergeben hinsichtlich der Anforderungen von Befunden oder des Ausfüllens der psychiatrischen Basisdokumentation (BADO). Dies könnte eine weitere Entlastung der Ärzte bezüglich ihrer in den letzten Jahren zunehmenden administrativen Aufgaben [4] bedeuten. Der DKI-Vorschlag der Vorbereitung des Arztbriefes durch nichtärztliche Fachangestellte erscheint dagegen nicht effizient: Denn auch das nichtärztliche Personal braucht Zeit und kostet Geld. Viele Tätigkeiten können vom Arzt aufgrund seiner Ausbildung und Kenntnis des Patienten besser und schneller erfolgen. Im Sinne eines wirklich effizienteren Personaleinsatzes sollten solche Tätigkeiten beim Arzt bleiben. Dies erscheint auch aus haftungsrechtlichen Gründen geboten, so z. B. beim Erstellen von Attesten, Rezepten und Entlassungsbriefen. Auch die Durchführung einer „Basisinformation im Rahmen der Patientenaufklärung“ - wie vom DKI vorgeschlagen - ist problematisch, angesichts der zunehmenden Arzthaftungsprozesse bezüglich einer unzureichenden Aufklärung [5]. Und ob die Patienten ihre Informationen überwiegend von nichtärztlichem Personal bekommen wollen und eine partizipative Entscheidungsfindung ohne den Arzt stattfinden soll, bleibt zu bezweifeln [6]. Der - politisch gewollte - Ausbau der Patientenberatung [7] wie auch die angestrebte Patientenbeteiligung gerade in der Psychiatrie [8] ohne die Mitwirkung des Arztes, ist wenig vorstellbar.

Generell ist die rechtliche Problematik erheblich. Ob die nichtärztlichen Mitarbeiter, die eine ärztliche Aufgabe übertragen bekommen, haftungsrechtlich tatsächlich „auf der sicheren Seite“ stehen, ist nicht garantiert. Letztendlich entscheiden die Gerichte rückwirkend, ob der nichtärztliche Mitarbeiter die übertragene Tätigkeit im Einzelfall tatsächlich hätte ausführen dürfen oder ob er wegen der besonderen Schwierigkeit im Rahmen eines Übernahmeverschuldens für Fehler haftet. Leitende Ärzte werden intensiv zu prüfen haben, ob eine Delegation ärztlicher Aufgaben - selbst da wo sie grundsätzlich rechtlich möglich sein sollte - wegen örtlicher Besonderheiten zu einer Haftung aus Organisationsverschulden führen kann. Besonders problematisch ist die Substitution ärztlicher Leistungen durch die Pflege, bei der das volle Haftungsrisiko an die dann selbstständig arbeitende Pflegekraft übergehen würde. Eine solche Substitution ist nach dem Pflege-Weiterentwicklungsgesetz (PWG) möglich. In welchem Umfang die Rechtsprechung diesem Paradigmenwechsel auch in problematischen Einzelfällen folgen wird, bleibt abzuwarten. Auf dem 111. Deutschen Ärztetag wurde jedenfalls erhebliche Kritik an der neuen Gesetzeslage laut.

Am Schluss bleibt daran zu erinnern, dass hinter all diesen Vorschlägen der DKG (neben dem Ärztemangel) nichts anderes steckt als das weitere Bemühen nach Kosteneinsparungen in den Kliniken. Anstelle einer Gesundheitsökonomie erleben wir weiter nur eine Ökonomisierung des Gesundheitssystems. Ziel der Umsetzung der Vorschläge der DKI-Studie ist natürlich, ärztliches d.h. teueres Personal einzusparen und durch billigere Personalgruppen zu ersetzen. Das bedeutet aber, dass künftig noch weniger Ärzte in Kliniken tätig sein werden. In letzter Konsequenz der Delegation und Substitution zahlreicher Aufgaben bleiben dem Arzt noch die fragmentierten Funktionen der raschen (Differenzial-)Diagnostik und (medikamentösen) Therapie. Er wird überwiegend in kritischen Situationen und in Bereitschaftsdiensten eingesetzt werden. Der Arzt wird - ungeachtet der in den letzten Jahren zunehmenden Leistungsverdichtung [9] und eines PsychPV-Erfüllungsgrades in den Kliniken von durchschnittlich 90\% [10] - in noch kürzerer Zeit noch mehr Patienten diagnostizieren und behandeln müssen. Ein ganzheitliches, ärztlich geleitetes „case management“ unter umfassender Berücksichtigung der biopsychosozialen Situation des Patienten (und seiner Angehörigen [11]) wird unter solchen Rahmenbedingungen noch weniger möglich sein. Hier stellt sich die Frage, ob dies tatsächlich eine lohnende Zukunft für psychiatrische Kliniken darstellt. Und: Eine längerfristige Lösung der Probleme im stationären Bereich wird sich durch die „Neuordnung von Aufgaben des ärztlichen Dienstes“ nicht ergeben, dazu bedarf es umfassenderer Ansätze [12-15].

\section{Literatur}

1 Deutsches Krankenhausinstitut. http://dki.comnetinfo.de/PDF/Neuord nung-Aerztlicher-Dienst_Langfassung.pdf

2 Hausner H, Hajak G, Spießl H. „Kooperation und Verantwortung“: Das neue Gutachten des Sachverständigenrates zur Entwicklung des Gesundheitswesens. Psychiat Prax 2007; 34: 365-366

3 Deutsche Krankenhausgesellschaft. http://www.dkgev.de/dkg.php/cat/ 38/aid/4880/title/DKG_zur_DKI-Studie_\%E2\%80\%9ENeuordnung_von Aufgaben_des_AErztlichen_Dienstes\%E2\%80\%9C

4 Putzhammer A, Senft I, Fleischmann H, Klein HE, Schmauß M, Schreiber $W$, Hajak G. Eine Tätigkeitsanalyse in psychiatrischen Versorgungskliniken. Nervenarzt 2006; 77: 372-384

5 Hausner H, Cording C, Hajak G, Spießl H. Aufklärung und Einwilligung in Psychiatrie und Psychotherapie. Psychiat Prax 2008; 35: 163-169

6 Spießl H, Spießl A, Cording C. Die „ideale“ stationär-psychiatrische Behandlung aus Sicht der Patienten. Psychiat Prax 1999; 26: 3-8

7 Kühn-Mengel H. Ausbau der Patientenberatung: bei psychischen Erkrankungen von besonderer Bedeutung. Psychiat Prax 2008; 35: 107

8 Reichart T, Kissling W, Scheuring E, Hamann J. Patientenbeteiligung in der Psychiatrie - eine kritische Bestandsaufnahme. Psychiat Prax 2008; 35: 111-121

9 Spießl H, Binder H, Cording C, Klein HE, Hajak G. Klinikpsychiatrie unter ökonomischem Druck. Dt Ärztebl 2006; 103: 2549-2552

10 Gebhardt RP, Schmidt-Michel PO, Kunze H. Evaluation der PsychiatriePersonalverordnung. Ausgewählte Ergebnisse aus der Umfrage 2005. Psychiat Prax 2008; 35: 99-100

11 Spießl H, Schmid R, Wiedemann G, Cording C. Unzufriedene Angehörige - Kunstfehler psychiatrischer Behandlung oder ökonomische Notwendigkeit? Psychiat Prax 2005; 32: 215-217

12 Roick C, Deister A, Zechner D, Birker T, König HH, Angermeyer MC. Das Regionale Psychiatriebudget: Ein neuer Ansatz zur effizienten Verknüpfung stationärer und ambulanter Versorgungsleistungen. Psychiat Prax 2005; 32: 177-184

13 Kunze H, Priebe S. Integrierte Versorgung - Perspektiven für die Psychiatrie und Psychotherapie. Psychiat Prax 2006: 33: 53-55

14 Kunze $H$. Personenbezogene Behandlung in psychiatrischen Kliniken und darüber hinaus - Gute Praxis und Ökonomie verbinden. Psychiat Prax 2007; 34: 150-153

15 Hambrecht M. Gemeindepsychiatrie im 21. Jahrhundert. Psychiat Prax 2007; 34: 262-265 